\title{
Herramientas para la investigación en Recuperación de Información: Karpanta, un motor de búsqueda experimental
}

\author{
Carlos García Figuerola \\ José Luis Alonso Berrocal \\ Ángel Francisco Zazo Rodríguez \\ Emilio Rodríguez Vázquez de Aldana \\ Universidad de Salamanca, Grupo REINA (España)
}

\subsection{Resumen}

Se presenta Karpanta, un motor de recuperación sumamente flexible, que implementa un gran número de algoritmos diferentes (más de 300), y que aísla el proceso de indización automática y resolución de consultas de las fases de análisis léxico y visualización. El código es sumamente simple y fácilmente modificable, dado que resuelve la totalidad de las operaciones mediante sencillas sentencias SQL, almacenando los datos en tablas relacionales. Karpanta es un paquete de código libre y abierto con licencia pública general (GPL) que puede ser utilizado, modificado y adaptado libremente por cualquier investigador; y se ha diseñado específicamente para constituir una herramienta de investigación en el área interdisciplinar de la Recuperación de la Información. De otro lado, Karpanta puede ser también usado con éxito operacionalmente, en entornos reales y para tareas reales como las que puedan darse en un Centro de Documentación.

Palabras clave: Recuperación de Información. Motores de búsqueda. Entornos digitales de investigación experimental. Karpanta.

\subsection{Abstract}

Karpanta, a search engine that implements a great number of different algorithms (more than 300), and that isolates the process of automatic indexing and resolving queries of the phases of lexical analysis and visualization, is presented. The code is very simple and easily modifiable, since it solves the totality of the operations by means of simple SQL sentences, storing the data in relational tables. Karpanta is free and open code with a GPL license that can be used, freely modified and adapted by any researcher; and has been implemented specifically as a research environment tool for the interdisciplinary field of Information 
Retrieval. On the other side, Karpanta also can be successfully used operationally for the real tasks that occur in a documentation centre.

Keywords: Information Retrieval. Search engines. Experimental research digital environments. Karpanta.

\section{Introducción}

La Recuperación de la Información (RI), aunque no es precisamente un área de investigación reciente, experimenta en los últimos tiempos un auge notable, debido a la disponibilidad cada vez mayor de documentos en formato electrónico. El desarrollo y generalización del uso de Internet ha puesto de manifiesto las carencias y los retos en este campo, de manera que son numerosos los grupos de investigadores que dirigen sus esfuerzos hacia estas materias. Uno de los campos de investigación en RI es la experimentación con diversos algoritmos, referentes a cualquiera de las fases o tareas que pueden darse en el proceso de recuperación. La investigación experimental en este campo, sin embargo, requiere, además de los conocimientos básicos necesarios, de una serie de herramientas o instrumentos que permitan la realización de experimentos. Entre tales instrumentos, podemos distinguir, a grandes rasgos tres grandes grupos: En primer lugar, se necesitan colecciones de documentos adecuadas, tanto por sus características documentales, como lingüísticas, e incluso de tamaño. Estas colecciones no sólo incluyen documentos, sino también baterías de preguntas o consultas, así como las correspondientes estimaciones de relevancia para las mismas. En segundo lugar, son necesarios programas que permitan indizar los documentos y resolver las consultas. Y, finalmente, se requieren medidas eficaces y aceptadas ampliamente por la comunidad científica, que permitan evaluar y comparar los resultados de los experimentos (Robertson, 1992; Su, 1992; Warner, 2000; Rijsbergen, 1979). Este trabajo se centra en la producción de herramientas comprendidas en el segundo punto, esto es, de programas capaces de indizar documentos y resolver consultas. Más concretamente, en la producción de un motor experimental de recuperación, que permita utilizar alternativamente y con facilidad distintos algoritmos.

\section{Motores experimentales de recuperación}

Básicamente, un motor de recuperación es un programa - o un conjunto de ellos - que es capaz de indizar documentos y de resolver o ejecutar consultas sobre tales documentos. Sus componentes pueden esquematizarse en cuatro módulos funcionales (Prager, 2000; Baeza-Yates, 2000):

1) Análisis léxico, es decir, la extracción de términos clave que han de representar el contenido de cada documento. Este análisis léxico puede consistir en un simple parsing o en procesos más complejos, como la lematización, el etiquetado semántico, etc.

Scire. $10: 2$ (jul.-dic. 2004) 51-62. 
2) Indización, o construcción de índices que permitan acceder a los documentos. Este proceso incluye la determinación del poder descriptivo de cada uno de los términos extraídos en la fase anterior.

3) Resolución de consultas, esto es, la estimación de la similitud entre una consulta y cada uno de los documentos de la colección.

4) Interfaz de usuario, que debe permitir a éste formular sus necesidades informativas, es decir, interactuar con el sistema. Esta interacción puede incluir elementos más complejos, como la realimentación de consultas, la selección de nuevos términos de búsqueda, la visualización de documentos o resúmenes de éstos, etc.

No obstante, suele entenderse que el corazón o núcleo, lo que realmente constituye un motor de recuperación, son los componentes 2 y 3 .

Existen, como es bien sabido, numerosos motores de recuperación operacionales, diseñados para trabajar en entornos reales. Cada uno implementa un modelo teórico y utiliza un juego de algoritmos fijo; deben atender a las necesidades del mundo real, como, por ejemplo, la velocidad en la ejecución; y, debido a esto - y también a razones comerciales en muchos casos - presentan una codificación específica destinada a resolver de la forma más eficiente posible sus tareas de una manera fija. Los motores experimentales, sin embargo, están destinados a la experimentación y no están coercionados por factores como la velocidad de ejecución. Su misión es admitir diversas vías de resolución de problemas, en distintos entornos y con distintos objetivos específicos. A grandes rasgos, las características deseables son las siguientes:

- Los componentes deben ser independientes entre sí, de manera que sea factible operar sobre parte de ellos, modificándolos, sin necesidad de tener que tocar el resto. Un motor experimental debería ser independiente, por ejemplo, del analizador léxico, de forma que fuera posible alterar el comportamiento de éste o incluso sustituirlo por otro con diferentes capacidades.

- El motor debe ser flexible como para incluir diversos algoritmos o aproximaciones a las tareas que debe resolver.

- Debe permitir la observación de resultados intermedios, incluso su manipulación o modificación.

- El código debe ser lo más sencillo y modular posible, para facilitar su modificación.

- En relación con el punto anterior, el código debe ser abierto y libremente disponible, así como estar escrito en versiones estándar de lenguajes estándar.

Lamentablemente, no existen muchos motores experimentales; y aún menos que cumplan estas condiciones. Existen motores experimentales que no son 
abiertos y que sólo los pueden utilizarlos investigadores que los diseñaron; y existen motores no experimentales que son utilizados - con grandes dificultades - por algunos grupos de investigación. Uno de los paradigmas de motor experimental, utilizado durante años por diferentes grupos de investigación, es el conocido SMART (Salton, 1971). Sin embargo, SMART, que ha prestado una ayuda inestimable a muchos investigadores y que es una excelente herramienta de experimentación, tiene cuatro inconvenientes. En primer lugar, está escasamente documentado en lo que se refiere a operación y estructura interna. Sorprendentemente, además de la magra documentación ofrecida por sus autores junto con el programa, el recurso más conocido es un breve curso de utilización básica (Paimans, 1999). Ni una ni otro cubren más que las capacidades más elementales del programa. En segundo lugar, sus componentes están fuertemente integrados, de manera que, por ejemplo, no es posible aislar el parser del motor propiamente. En tercer lugar, el código es prolijo y complejo, lo que hace difícil su modificación. En cuarto lugar, presenta, además, algunos problemas de portabilidad.

\section{Objetivos}

El objetivo de este trabajo es, pues, la realización de un motor de recuperación experimental. Sus características de partida son: a) consta tan sólo de dos componentes - un indizador y un estimador de similitud entre consulta y documentos -; b) cada uno de estos dos componentes debe aceptar como entrada los resultados de componentes previos, y producir, en su caso, salidas que puedan ser usadas por componentes o procesos posteriores; c) el motor debe permitir la utilización, a elección por el usuario, de diferentes algoritmos, e incluir la mayor cantidad posible de éstos; d) el motor debe permitir la inclusión fácil de nuevos algoritmos; e) el motor permitirá la inspección y manipulación, de resultados intermedios; f) el motor debe estar escrito en un código lo más breve y simple posible, fácil de modificar, e incluso fácil de utilizar como modelo para la realización de otros programas o implementaciones; y g) dado su carácter experimental, las características de flexibilidad, legibilidad y sencillez de código deberían primar sobre las de eficacia (especialmente velocidad).

Teniendo en cuenta todo esto, se ha considerado una opción razonable la utilización de tablas relacionales para almacenar la información extraída de los documentos, y de sentencias SQL para la realización de las operaciones necesarias. Ambas técnicas están lo suficientemente estandarizadas como para poder ser modificadas fácilmente por cualquiera. La estructura relacional y el SQL son especialmente potentes e intuitivos y facilitan la comprensión y ejecución de las operaciones necesarias.

Scire. $10: 2$ (jul.-dic. 2004) 51-62. 


\section{El modelo vectorial}

El modelo teórico más difundido en RI es el llamado modelo vectorial (Salton, 1983). Básicamente, según éste, cada documento es representado por un vector de $n$ elementos donde $n$ es el número de términos posibles en toda la colección de documentos, y cada elemento del vector, en consecuencia, corresponde a cada uno de tales términos. Los elementos del vector, por otra parte, consisten en un valor numérico que trata de expresar la importancia o peso del término en cuestión dentro del documento. Es obvio que un mismo término en documentos diferentes debe tener pesos diferentes.

Las consultas se tratan igual que los documentos, y se representan igualmente mediante un vector de pesos. Así, la resolución de una consulta consiste simplemente en la computación de alguna función de similitud entre el vector consulta y cada uno de los vectores de los documentos. Este tratamiento tiene dos ventajas importantes: en primer lugar, permite que las consultas se hagan en lenguaje natural, y pueden ser del tamaño que se desee; en segundo lugar, dado que el resultado de la función de similitud no tiene porqué ser binario, es posible establecer una graduación o escala en las respuestas a las consultas.

La clave de todo el sistema reside en lo bien que los documentos $-\mathrm{y}$ las consultas - estén representados a través de los vectores; y ésto depende a su vez de dos factores: la determinación de los términos que se extraen de cada documento, y la forma en que se estiman o calculan los pesos de cada término en cada documento. El primero de estos factores (análisis léxico) queda fuera de nuestro objetivo, pero debe indicarse la conveniencia de aislar esta parte, de forma que, a afectos de experimentación, pueda operarse sobre ella libremente. El segundo factor (el cálculo de los pesos) constituye uno de los elementos centrales de nuestro trabajo.

\subsection{El peso de los términos}

La estimación del peso de cada término en cada documento puede hacerse de diversas formas, y de hecho se ha propuesto una buena cantidad de ellas. El cálculo de los pesos se efectúa a partir de dos factores (Harman, 1992): la frecuencia de cada término en cada documento, y un elemento conocido como IDF (Inverse Document Frequency). Adicionalmente, suele aplicarse algún factor de normalización que permita soslayar las diferencias de tamaño de los documentos. El IDF es una función inversamente proporcional a la frecuencia del término en toda la colección de documentos o base de datos. La hipótesis de partida es que los términos que aparecen en muchos documentos tienen un poder discriminatorio pobre, y viceversa. El peso, en consecuencia, podría estimarse a partir de una ecuación genérica:

Scire. $10: 2$ (jul.-dic. 2004) 51-62. 
peso_término_en_documento $=\frac{\text { frecuencia_término_en_documento } \times I D F}{\text { factor_de_normalización }}$

Cada uno de los tres elementos que intervienen en la ecuación puede ser calculado de distintas formas, lo cual da lugar a un gran número de variantes o esquemas de pesado. Usualmente, un esquema se representa mediante tres letras, cada una de las cuales identifica la forma en que se han calculado, respectivamente, la frecuencia del término en el documento, el IDF del término y el factor de normalización.

\subsection{Esquemas de peso}

Entre las muchas posibilidades existentes, se relacionan a continuación las formas más utilizadas de calcular cada uno de estos tres elementos. La frecuencia del término en el documento se calcula con las siguientes fórmulas:

- $\quad$ ninguna $(\mathrm{n})=n_{t d} d$

- $\quad$ binaria $(\mathrm{b})=1$

- $\max -\operatorname{norm}(\mathrm{m})=\frac{n_{t d}}{\max _{n d}}$

- aug-norm (a) $=0.5+0.5 \frac{n_{t d}}{\max _{n d}}$

- $\quad$ square $(\mathrm{s})=n_{t d}^{2}$

- $\quad \log (\mathrm{I})=\ln \left(n_{t d}\right)+1$

- double-log (d) $=\ln \left(\ln \left(n_{t d}\right)+1\right)+1$

- length-norm $(\mathrm{t})=\frac{\ln \left(n_{t d}\right)+1}{\ln \left(a v g_{n d}\right)+1}$

donde:

- $n_{t d}$ es el número de veces que el término $t$ aparece en el documento $d$

- $\max _{n d}$ es el número de veces que aparece el término más frecuente en el documento $d$

- $a v g_{n d}$ es la media de todas las frecuencias de términos en el documento $d$

El IDF se calcula normalmente con las siguientes fórmulas:

- $\operatorname{ninguna}(n)=1$

- $\quad$ tidf $(\mathrm{t})=\ln \left(\frac{N}{n_{t}}\right)$

- $\operatorname{prob}(\mathrm{p})=\ln \left(\frac{N-n_{t}}{n_{t}}\right)$

$-\quad \operatorname{frec}(\mathrm{f})=\frac{1}{n_{t}}$ 
- square (s) $=\left(\ln \frac{N}{n_{t}}\right)^{2}$

donde:

- $N$ es el número de documentos en la colección

- $n_{t}$ es el número de documentos en que aparece el término $t$

Finalmente, se usan para el factor de normalización:

donde:

- $n$ es el número de términos únicos en el documento $d$

- $\quad$ ninguno $(\mathrm{n})=1$

- $\operatorname{coseno}(\mathrm{c})=\sqrt{\sum_{i=1}^{n} P_{i d}{ }^{2}}$

- $\quad \operatorname{sum}(\mathrm{s})=\sum_{i=1}^{n} p_{i d}$

- $\quad$ fourth (f) $=\sum_{i=1}^{n} p^{4}{ }_{i d}$

- $\max (\mathrm{m})=\max _{p d}$

- $p_{i d}$ es el peso (frecuencia x IDF) del término $i$ en el documento $d$

- $\max _{p d}$ es el valor más alto de peso sin normalizar en el documento $d$

\section{Implementación}

Se puede almacenar un fichero invertido procedente de una colección de documentos en una tabla, de manera que, a partir de ahí, es posible calcular los pesos de términos así como las similitudes entre documentos y consultas. Un fichero invertido, en su forma más básica, no es más que una serie de entradas, una para cada término de la colección de documentos, para el que se almacena una lista de los documentos en que aparece. Esta estructura puede mapearse simplemente a una tabla con dos campos: término y clave de documento; pero nada impide, naturalmente, añadir más columnas para información vinculada a cada una de las parejas término-documento, como frecuencia, offset del documento en que aparece el término, etc.

A partir de aquí es sencillo obtener información adicional para calcular pesos: el número de documentos en la colección - select count(documento) from tabla; -, el número de documentos en que aparece cada término - select termino, count(documento) from tabla group by termino;-, etc. (Grossman, 1996; Grossman, 1997). 


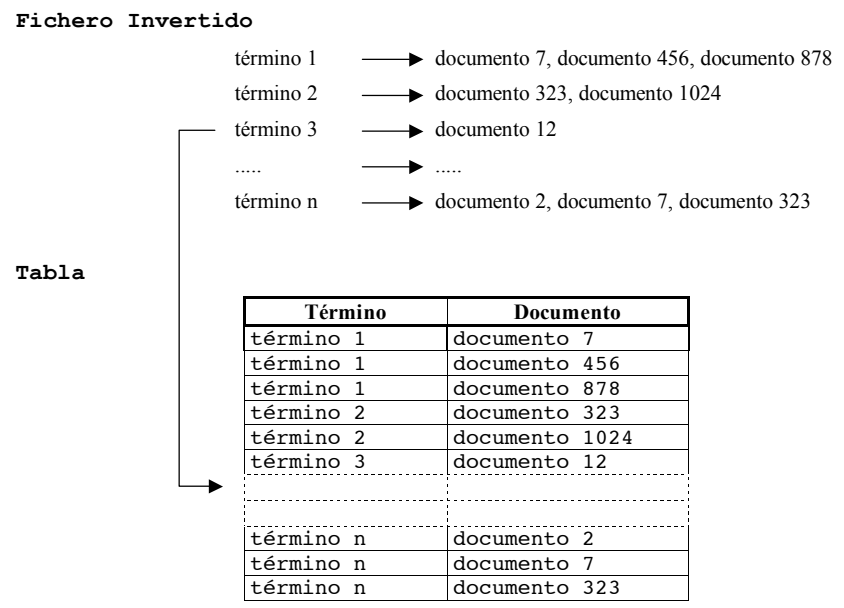

Fig. 1. Mapeo de fichero invertido a tabla simple

\subsection{La librería SQlite}

Para la realización de los programas hemos optado como motor SQL por la librería SQlite (Hipp, 2003). No se trata de una librería interfaz con un servidor $\mathrm{SQL}$, sino que ella misma contiene el servidor. Esto proporciona una mayor autonomía, al no depender de terceros programas. SQlite tiene algunas características generales notables: a) es una librería de código libre; b) el código es completamente portable, de forma que se puede recompilar prácticamente en cualquier plataforma - en Linux hemos compilado con GNU C (v. 2.9.54), en MS-DOS/ Windows con Mingw32 (Minimalist, 2003) sin necesidad de ningún reajuste-; c) el conjunto de sentencias SQL que reconoce es reducido, pero completamente estándar, lo cual significa que esas mismas sentencias funcionarán con cualquier otro servidor SQL; d) el conjunto de funciones regulares y agregadas de que dispone es muy reducido, aunque implementa mecanismos para añadir funciones de usuario - por ejemplo, para obtener logaritmos o raíces cuadradas -; e) el modo de pasar sentencias SQL es sencillo y claro, lo que facilita enormemente la legibilidad y modificabilidad del código del programa; f) la velocidad de ejecución, aunque no es factor prioritario, es razonable; y g) el consumo de memoria es bajo, aunque el de disco, sin embargo, es alto.

\subsection{Estructura básica de la base de datos}

La base de datos consta de varias tablas. Algunas son temporales, y desaparecen una vez calculados los pesos, y dependen del esquema de cálculo concreto adoptado en cada ocasión. Consta también de algunas vistas (para el procesa- 
miento de consultas), aunque se ha procurado evitar su uso, dada la poca eficacia con que las gestiona SQlite. Las tablas básicas son:

- terminos(termino char(35), documento char(35), veces double)

- pesos_def(termino char(35), documento char(35), peso double)

En realidad, SQlite no distingue tipos de datos, sino que almacena todos como secuencias de caracteres con un byte 0 como terminador. Pero, por razones de portabilidad se han establecido estos tipos. De otro lado, el número de veces que un término aparece en un documento - que en principio debería ser un entero - se establece como doble. La razón es que ésto permite que el parser u otro proceso previo aplique, si se desea, algún tipo de coeficiente que prime de distinta forma los términos en función de distintos criterios (lugar del documento donde aparece, tipografía, función sintáctica, etc.). Para la resolución de consultas, en realidad, sólo es precisa la tabla pesos_def, pero la tabla Términos es preciso conservarla para posibles recalculados de pesos posteriores.

\subsection{Entrada de datos}

El motor de recuperación espera recibir como entrada lo siguiente:

"término","documento", número de ocurrencias en documento

$\mathrm{y}$, tal cual, lo almacena en una tabla. Sobre la información almacenada en esta tabla se harán las operaciones posteriores. El programa no efectúa ningún chequeo ni ninguna otra operación previa sobre los datos de entrada. Ésto significa que cosas como la normalización de caracteres, eliminación de palabras vacías, etc., son responsabilidad del parser o de cualesquiera otros procesos intermedios que se quieran añadir.

\subsection{Cálculo de pesos}

El cálculo de pesos se efectúa en tres fases, una para cada componente del peso (Salton, 1988). Cada una de estas fases termina con una tabla temporal que recoge el componente calculado y que es usada en la fase siguiente. La tercera y última fase finaliza con la consecución de la tabla pesos_def, con lo que esas tablas temporales dejan de ser necesarias. No hay atajos, en aras de una mayor claridad del código.

\subsubsection{La frecuencia del término en el documento}

El mecanismo concreto depende del esquema de cálculo aplicado, pero, en general, se resuelve con un select sobre la tabla términos que contiene los datos originales de entrada. Por ejemplo, para el esquema $1(\log )$

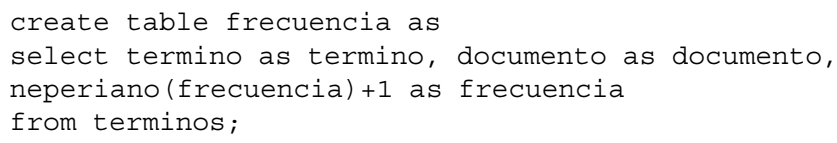

Scire. $10: 2$ (jul.-dic. 2004) 51-62. 
Con algunos esquemas que utilizan parámetros como la frecuencia máxima en el documento, es preciso algún paso intermedio que calcule tales elementos.

\subsubsection{El IDF y el peso sin normalizar}

El IDF es el mismo para cada término, independientemente del documento en el que éste último aparezca; de manera que el resultado del cálculo del IDF podría ser una tabla con los campos término e idf. El peso sin normalizar, por otra parte, es el resultado de multiplicar la frecuencia del término por el IDF; así, obtenido el IDF, puede obtenerse en la misma fase el peso sin normalizar. El producto final de esta fase es una tabla con los campos término, documento y peso. Por ejemplo, para calcular el IDF según el esquema $\mathrm{f}$ (frec) se puede utilizar el siguiente algoritmo:

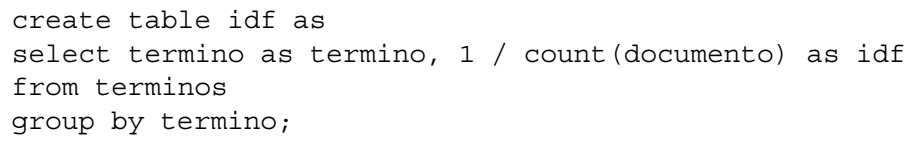

Una vez obtenido el IDF, sólo nos queda calcular el peso sin normalizar y almacenarlo en una tabla:



\subsubsection{El factor de normalización y pesos definitivos}

Esta fase, última por lo que se refiere a los documentos, requiere el cálculo de un factor de normalización, y la posterior división del peso sin normalizar - que acabamos de almacenar en la tabla pesos - por dicho factor. El factor de normalización, por otra parte, es único para cada documento. A modo de ejemplo, para normalizar mediante el esquema c (cos) se puede proceder así:

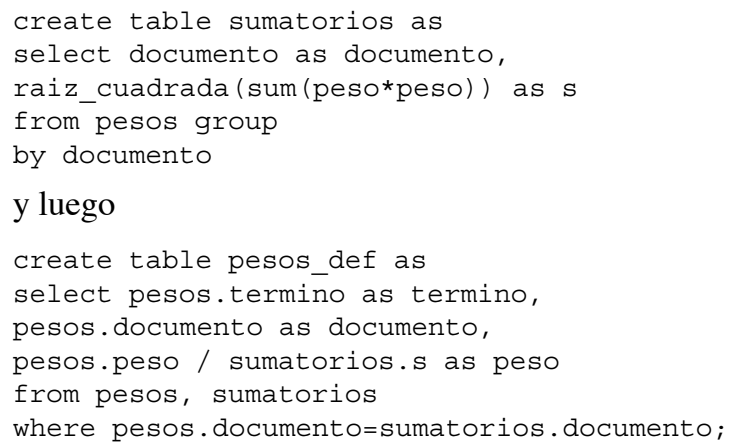

Scire. $10: 2$ (jul.-dic. 2004) 51-62. 
Hasta aquí, se han obtenido los pesos de los términos de los documentos, con lo que sólo se necesitará la tabla pesos_def y la que contiene los datos originales, términos, de manera que es posible eliminar las demás. La tabla pesos_def, por otra parte, requiere, para resolver más rápidamente las consultas, un índice de término.

\subsection{Pesos de las consultas}

Los pesos de los términos de las consultas se calculan aplicando esquemas que no tienen porqué ser iguales. Sin embargo, dado que se resuelve una sola consulta cada vez, el volumen de datos a manejar es considerablemente menor. Además, las consultas suelen ser mucho más cortas que cualquier documento, usualmente 2 ó 3 palabras. La solución adoptada aquí se basa en el uso de vistas. Se comentó más arriba que SQlite tiene un tratamiento poco eficaz de éstas. Sin embargo, cuando las vistas tienen que manejar una cantidad pequeña de datos, el rendimiento es aceptable. Esto permite, dejar construidas las vistas necesarias para calcular los pesos de los términos, al tiempo que se indiza la colección de documentos. En el momento de la consulta, estas vistas se ejecutan, y se obtienen los pesos correspondientes.

\subsection{Resolución de consultas y salida}

Para la resolución de consultas se ha habilitado un programa independiente que opera sobre la base de datos y sus correspondientes tablas y vistas. Al igual que se ha hecho con los documentos, el programa de búsqueda espera recibir el resultado del análisis léxico de la consulta a resolver, en el mismo formato. Esta entrada pasa a una tabla, sobre la que se ejecutarán las vistas definidas en el proceso de indización de los documentos, de acuerdo con el esquema de pesos que se haya especificado. De hecho, dichas vistas son llamadas directamente con la sentencia SQL que resuelve la consulta, de manera que dichas vistas se ejecutan calculando los pesos de los términos de la consulta y, acto seguido, calculando la similitud entre la consulta cada uno de los documentos. En realidad, sólo se calcula la similitud con los documentos que tienen en común al menos un término con la consulta. Con la misma sentencia se ordenan de forma decreciente dichas similitudes y sus documentos asociados. A partir de aquí, sólo queda producir una salida con las claves de los documentos recuperados y sus coeficientes de similitud. Puesto que el objetivo principal de este motor es la experimentación, uno de los formatos de salida posibles es acorde con el conocido programa treceval, el cual, como es conocido, calcula las medidas más utilizadas para evaluar la eficiencia en la recuperación.

\section{Conclusiones}

Se ha mostrado la estructura funcionamiento de un motor de recuperación de información diseñado para la investigación experimental. Este motor es de Scire. $10: 2$ (jul.-dic. 2004) 51-62. 


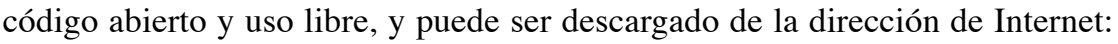
http://reina.usal.es/materiales.htm.

\section{Referencias}

Baeza-Yates, R.; Ribeiro-Neto, B. (2000). Modern Information Retrieval. Harlow: Addisson-Wesley, 2000.

Grossman, D. A.; Lundquist, C.; Reichart, J.; Holmes, D.; Chowdhury, A. Y Frieder, O. (1996). Using Relevance Feedback within the Relational Model for TREC-5. // Vorhees, E. M.; Harman, D. (eds.). The Fifth Tex Retrieval Conference (TREC-5). Gaithersburg, Maryland: NIST, 1996..

Grossman, D.; Frieder, O.; Holmes, D. y Roberts, D. (1997). Integrating Structured Data and Text: A Relational Approach. // JASIS. 48:2 122-132.

Harman, D. (1982). Ranking Algorithms. // Frakes, W. B. Y Baeza-Yatez, R. (eds.). Information Retrieval. Data Structures and Algorithms. Upper Sadle River NJ: Prentice-Hall, 1982. 363-392.

Hipp, R. (2003). SQlite: An Embeddable SQL Database Engine. URL: <http:// www.SQlite.org>. Consultado: 31-10-2003.

Minimalist GNU For Windows. URL: <http://www.mingw.org>. Consultado: 31-102003.

Paimans, H. (1999). SMART. Tutorial for beginners. URL: <http://pi0959.uvt.nl/Paai/ Onderw/Smart/hands-on-tekst.html>. Consultado: 31-10-2003.

Prager, J.; Brown, J.; Radev, D.; Czuba, K. (2000). One Search Engine or Two for Question Answering. // TREC-9. (2000) 235-240.

Rijsbergen, C. J. van (1979). Information Retrieval. London: Butterworths, 1979.

Robertson, S. E.; Hancock-Beaulieu, M. H. (1992). On the Evaluation of the IR Systems. // Information processing and Management. 28 (1992) 457-466.

Salton, G. (1971). The SMART Retrieval System. Experiments in Automatic Document Processing. Englewood Cliffs, NJ: Prentice Hall, 1971.

Salton, G.; Buckley, C. (1988). Term-weighting approaches in automatic text retrieval. // Information processing \& Management. 24:5 (1988) 513-523.

Salton, G. (1989). Automatic Text Processing: the Transformation, Analysis and Retrieval of Information by Computer. Reading MA: Addison-Wesley, 1989.

Singhal, A.; Buckley, C.; Mitra, M. (1996). Pivoted document length normalization. // Proceedings of the 19th ACM Conference on Research and Development in Information Retrieval: SIGIR 96. (1996) 21-29.

Su, L. T. (1992). Evaluation Measures for Interactive Information Retrieval. // Information Processing and Management. 28 (1992) 503-516.

Warner, J. (2000). In the Catalalogue Ye Go for Men: Evaluation Criteria for Information Retrieval. // Aslib Procs. 52:2 (2000) 76-82. 